NOTA CIENTÍFICA

\title{
CONTROLE QUÍMICO DA FERRUGEM ASIÁTICA DA SOJA NA REGIÃO SUL DO PARANÁ
}

\section{CHEMICAL CONTROL OF ASIAN SOYBEAN RUST IN SOUTH PARANÁ STATE}

\author{
Emerson Fábio dos REIS ${ }^{1}$ \\ Vismar da Costa LIMA NETO² \\ Cláudia Vieira GODOY ${ }^{3}$ \\ Cleber Teixeira ROSA ${ }^{4}$ \\ Hailton Ezequiel CASTANHO 5 \\ Nilson Gonçalves VICENTE ${ }^{6}$
}

\begin{abstract}
RESUMO
A ferrugem asiática da soja, causada pelo fungo Phakopsora pachyrhizi, é um problema recente no Brasil, podendo causar queda prematura das folhas e conseqüente redução de produtividade. Tendo em vista a inexistência de cultivares resistentes o controle químico é, atualmente, a mais importante alternativa para controle da doença. Este trabalho teve como objetivo testar dois princípios ativos fungicidas e 5 épocas de aplicação para controle da ferrugem da soja. Os experimentos foram realizados em Palmeira-PR, durante o período de novembro de 2003 a maio de 2004, nas cultivares CD 205 e BRS 133. Os ingredientes ativos testados foram: tetraconazol ( $50 \mathrm{~g}$ i.a. ha-1 $)$ e pyraclostrobina $\left(66,5 \mathrm{~g}\right.$ i.a. ha $\left.{ }^{-1}\right)+$ epoxiconazol $\left(25 \mathrm{~g}\right.$ i.a. ha- $\left.{ }^{-1}\right)$. Foram comparadas aplicações únicas nos estádios V7 (com sintomas iniciais da doença), V9, R1, R2 e R5.1 à duas aplicações nos estádios R2 e R5.1. O delineamento experimental utilizado foi blocos ao acaso com quatro repetições e número variável de tratamentos em cada ensaio (9 tratamentos na cultivar BRS 133 e 7 na CD 205). A área colhida de cada parcela foi de 5,4 $\mathrm{m}^{2}$. Os tratamentos contendo apenas triazol ou mistura de estrobilurina + triazol demonstraram efeito protetor e curativo apresentando resultados similares no controle da doença. A aplicação única dos fungicidas tetraconazol e pyraclostrobina + epoxiconazol mostraram maior eficiência quando aplicados com baixa severidade do fungo. Entretanto, verificou-se que as primeiras aplicações dos fungicidas em V7 para a cultivar BRS 133 e, R1 para CD 205, resultou em menor evolução da severidade da ferrugem.
\end{abstract}

Palavras-chave: doenças da soja; Glycine Max; Phakopsora pachyrhizi.

\begin{abstract}
Asian soybean rust, caused by the fungus Phakopsora pachyrhizi, is a recent problem on soybean in Brazil. Affected plants shed their leaves prematurely leading to crop losses. The present work was undertaken with the aim of testing two fungicidal active ingredients and 5 application times. The trials were established in Palmeira, from November 2003 to May 2004, with the cultivars CD 205 and BRS133. The active ingredients (a.i) assayed were tetraconazol ( $50 \mathrm{~g}$ a.i. ha $\left.{ }^{-1}\right)$, and pyraclostrobina ( $66.5 \mathrm{~g}$ a.i. $\mathrm{ha}^{-1}$ ) + epoxiconazol ( $25 \mathrm{~g}$ a.i. ha $\left.{ }^{-1}\right)$. One application at stages V7 (initial disease symptoms), V9, R1, R2 and R5.1 was compared with one and two applications at stages R2 and R5.1. Trials were carried out using randomized block designs with four replicates and variable numbers of treatments per trial (nine treatments on the cv. BRS 133 and seven on the cv. CD 205). The harvest area of each plot was $5,4 \mathrm{~m}^{2}$. The treatments with triazol and the mixture of estrobirulina + triazol showed protective and curative efficiency on Asian soybean rust with similar disease control. The treatment with tetraconazol and pyraclostrobina + epoxiconazol showed higher efficiency when applied under low fungal severity. However, it was verified that the first application of fungicide at stage V7 on cv. BRS 133 and R1 on cv. CD 205 resulted in a slower evolution of the rust severity.
\end{abstract}

Key-words: Glycine max; Phakopsora pachyrhizi; soybean diseases.

\footnotetext{
${ }^{1}$ Engenheiro agrônomo, Arysta Lifescience do Brasil. Rua Conrado Schiffer, n. 60, Apto 202, Bloco 4. Ponta Grossa PR, CEP 84050-280. E-mail: emersonfreis@terra.com.br.

2Professor, Universidade Federal do Paraná, Rua dos Funcionários, n. 1540, Juvevê. Curitiba PR. CEP 80035-050. E-mail vismar@terra.com.br (autor para correspondência)

${ }^{3}$ Fitopatologista, EMBRAPA - Londrina PR, Rodovia Carlos João Strass. Caixa Postal 231. Londrina PR. CEP 850001-970. E-mail: godoy@cnpso.embrapa.br

${ }^{4}$ Engenheiro agrônomo, Arysta Lifescience do Brasil. Avenida Sete de Setembro, n. 4881, sala 141 - Curitiba PR. CEP 80240-120.E-mail: clebertexeira@terra.com.br

${ }^{5}$ Engenheiro agrônomo, Arysta Lifescience do Brasil. Avenida Sete de Setembro, n. 4881, sala 141 - Curitiba PR. CEP 80240-120. haitoncastanho@terra.com.br

${ }^{6}$ Engenheiro agrônomo, Arysta Lifescience do Brasil. Avenida Sete de Setembro, n. 4881, sala 141 - Curitiba PR. CEP 80240-120. nilsongv@terra.com.br
} 


\section{INTRODUÇÃO}

A ferrugem asiática, causada por Phakospora pachyrhizi H. Sydow \& P. Sydow, foi identificada no Brasil pela primeira vez no final da safra 2000/01, através de dois trabalhos de detecção da doença no Paraná, realizados por YORINORI et al. (2001) e JACCOUD FILHO et al. (2001).

Os sintomas iniciais da doença consistem de pequenas lesões foliares, de coloração castanha a marrom-escura. Na face inferior da folha pode-se observar soros urediniais liberando os urediniósporos. As lesões tendem para o formato angular com 2 a $5 \mathrm{~mm}$ de diâmetro e, embora não seja comum, podem ocorrer nos pecíolos, vagens e caules (SINCLAIR E HARTMAN, 1999). Plantas severamente infectadas apresentam desfolha precoce, comprometendo a formação e o peso final dos grãos (YANG et al., 1991). Os danos causados pela ferrugem asiática em outros países produtores, onde a doença ocorre a mais tempo, são acentuados podendo causar reduções de até $80 \%$ na produtividade (SINCLAIR e HARTMAN, 1999).

Na safra 2001/02 o patógeno encontravase disseminado pelas principais regiões produtoras do Brasil causando perdas na ordem de $112.000 \mathrm{t}$, equivalentes a US\$24,70 milhões e as perdas de rendimento variaram de $30 \%$ a $75 \%$ (YORINORI et al., 2002).

A obtenção de cultivares de soja resistentes à ferrugem asiática tem sido um desafio para pesquisa. Conseqüentemente, o controle químico tem sido a medida de controle mais viável em outros países onde a ferrugem asiática ocorre. No Brasil, devido a recente introdução da doença, há carência de informações, em nível de lavoura, em relação à época ideal de aplicação, à eficiência dos produtos e número de aplicações necessárias para um controle econômico.

Os trabalhos disponíveis na literatura sobre controle químico da ferrugem são limitados aos testes de eficiência, e os fungicidas registrados para controle da ferrugem da soja encontram-se agrupados quanto à eficiência por meio de resultados de uma rede de ensaios (GODOY, 2005). Segundo GODOY e CANTERI (2004) os princípios ativos tebuconazol, difenoconazol, azoxystrobina e pyraclotrobina + epoxiconazol reduziram a infecção quando aplicados na ausência de sintomas, durante o período de incubação da ferrugem. No entanto, nenhum dos produtos avaliados impediu o desenvolvimento da doença, atuando de forma erradicante. Todos os produtos testados reduziram a severidade da doença e a viabilidade dos urediniósporos.

Faz-se necessário, portanto, estudar metodologias para otimizar o controle químico que minimizem os prejuízos com produtos e que apresentem características desejáveis, especialmente aquelas associadas ao amplo espectro de ação, baixa toxicidade, eficiente poder curativo e maior período residual. Desta forma, o presente trabalho teve como objetivo estudar a eficiência de diferentes princípios ativos no controle da ferrugem asiática da soja, em função do estádio fenológico da planta e número de aplicações.

\section{METODOLOGIA}

Os experimentos foram realizados no município de Palmeira/PR, de novembro de 2003 a maio de 2004, sendo utilizado as cultivares CD 205 e BRS 133. Os ingredientes ativos (i.a.) testados foram: tetraconazol ( $50 \mathrm{~g}$ i.a. ha $\left.{ }^{-1}\right)$, nome comercial Eminent e pyraclostrobina $\left(66,5 \mathrm{~g}\right.$ i.a. ha $\left.{ }^{-1}\right)+$ epoxiconazol ( $25 \mathrm{~g}$ i.a. ha-1 ${ }^{-1}$, nome comercial Opera. Foram comparadas aplicações únicas nos estádios V7 (com sintomas iniciais da doença), V9, R1, R2 e R5.1 e duas aplicações nos estádios R2 e R5.1.

Os tratamentos foram dispostos segundo o delineamento experimental em blocos ao acaso e quatro repetições, sendo nove tratamentos com a cultivar BRS 133 e sete tratamentos com a cultivar CD 205. Cada unidade experimental constituiu-se de 5 linhas espaçadas de $0,45 \mathrm{~m}$ e $7 \mathrm{~m}$ de comprimento representando área total de $15,75 \mathrm{~m}^{2}$. Como área útil, utilizou-se as 2 linhas centrais e descartou-se $0,5 \mathrm{~m}$ nas extremidades, totalizando-se $5,4 \mathrm{~m}^{2}$.

Os produtos foram aplicados utilizandose pulverizador costal pressurizado com $\mathrm{CO}_{2}$, com pressão de $3 \mathrm{lbs} \mathrm{pol}^{-2}$, barra de $2 \mathrm{~m}$ contendo 4 bicos, espaçados a 0,5 m, tipo leque (XR 11002 / Teejet) numa vazão de $200 \mathrm{~L} \mathrm{ha}^{-1}$.

As avaliações foram realizadas em intervalos de 8 dias após a primeira aplicação. Para isso, tomou-se 10 plantas ao acaso em cada parcela, e atribuiu-se um valor médio em porcentagem de área foliar lesionada nos folíolos obtidos nos terços superior, médio e inferior. Adotando-se a escala diagramática para avaliação da ferrugem da soja proposta por CANTERI e GODOY (2003).

A desfolha foi avaliada no estádio R7 (início da maturação) através de avaliação visual, atribuiu-se um valor em porcentagem de desfolha para cada tratamento. Como rendimento, avaliou-se a produtividade e peso de mil grãos (PMG). Para isso, foram colhidas manualmente e trilhadas. Após a pesagem dos grãos obtidos em cada parcela e aferida a umidade, esses foram corrigidos para $13 \%$ de umidade e transformado em $\mathrm{kg} \mathrm{ha}^{-1}$ utilizando a fórmula (correção da umidade $=100-$ umidade da amostra / $87 \mathrm{x}$ peso da amostra) e peso de mil grãos em gramas.

Os dados foram submetidos à análise de variância utilizando o programa ARM7 ao constatar diferenças significativas das médias entre os tratamentos, foram comparadas entre si pelo teste de Tukey a $5 \%$ de probabilidade. 
REIS, E.F. et al. Controle químico da ferrugem asiática...

\section{RESULTADOS E DISCUSSÃO}

Observou-se que em todas as cultivares utilizadas nos ensaios apresentaram suscetibilidade ao fungo $P$. pachyrhizi e a utilização da pulverização com fungicidas do grupo dos triazóis e a mistura de estrobilurina + triazol influenciaram significativamente na evolução da doença (Tabelas 1 e 3).

Para a cultivar BRS 133, a severidade máxima observada na testemunha foi de $11,25 \%$ no estádio R5.3 (Tabela 1). Embora a doença tenha iniciado no estádio vegetativo, não foi suficiente para causar redução significativa na produtividade (Tabela 2). A baixa precipitação pluviométrica após a constatação dos sintomas iniciais durante o ciclo da cultura, provavelmente não permitiu o desenvolvimento da doença a níveis mais elevados.

TABELA 1 - Severidade da ferrugem asiática sobre a cultivar BRS 133, safra 2003/04, Palmeira-PR.

\begin{tabular}{|c|c|c|c|c|c|}
\hline \multirow[t]{2}{*}{ Tratamento } & \multicolumn{5}{|c|}{ Severidade (\%) } \\
\hline & Aplicação $^{1}$ & Prévia & $21 \mathrm{DAA}^{*}$ & $29 \mathrm{DAA}^{*}$ & $41 \mathrm{DAA}^{*}$ \\
\hline Testemunha & - & $0,16 \mathrm{a}$ & 5,25 cde & $10,00 \mathrm{a}$ & $11,25 \mathrm{a}$ \\
\hline Tetraconazole125EW & V7 & $0,25 a$ & 4,18 e & $5,88 d$ & $6,38 d$ \\
\hline Tetraconazole125EW & V9 & $0,19 a$ & $4,75 \mathrm{de}$ & $6,88 \mathrm{bcd}$ & $7,38 \mathrm{~cd}$ \\
\hline Tetraconazole125EW & $\mathrm{R} 2$ e $\mathrm{R} 5.1$ & $0,17 \mathrm{a}$ & $6,33 \mathrm{bc}$ & $6,55 \mathrm{~cd}$ & $7,25 \mathrm{~cd}$ \\
\hline Tetraconazole 125EW & R5.1 & $0,24 a$ & $8,00 \mathrm{a}$ & $10,00 \mathrm{a}$ & $10,63 \mathrm{a}$ \\
\hline Pyraclostrobin+epoxiconazole & V7 & $0,19 a$ & $4,98 \mathrm{de}$ & $6,00 \mathrm{~d}$ & $6,88 \mathrm{~cd}$ \\
\hline Pyraclostrobin+epoxiconazole & V9 & $0,17 \mathrm{a}$ & $5,60 \mathrm{~cd}$ & $6,93 \mathrm{bcd}$ & $7,70 \mathrm{bcd}$ \\
\hline Pyraclostrobin+epoxiconazole & R2 e R5.1 & $0,27 \mathrm{a}$ & $6,43 \mathrm{bc}$ & $7,63 \mathrm{bc}$ & $8,25 \mathrm{bc}$ \\
\hline Pyraclostrobin+epoxiconazole & R5.1 & $0,22 \mathrm{a}$ & $6,93 \mathrm{~b}$ & $8,13 b$ & $9,00 \mathrm{~b}$ \\
\hline CV $(\%)$ & & 49,16 & 11,09 & 9,83 & 9,08 \\
\hline
\end{tabular}

Observa-se que os tratamentos com fungicidas apresentaram resultados significativos na redução da desfolha (Tabela 2). Todavia, não favorecendo diferença significativa no rendimento da cultura, quando comparado aos demais tratamentos (Tabela 2). No entanto, foram observadas diferenças de produtividade de $948 \mathrm{~kg} \mathrm{ha}^{-1}$ quando comparado à aplicação do tetraconazol no estádio V7 e a testemunha.

TABELA 2 - Percentagem de desfolha, peso de mil grãos e produtividade da cultivar BRS 133, após aplicação de fungicidas em diferentes estádios fenológicos, safra 2003/04, Palmeira-PR.

\begin{tabular}{|c|c|c|c|c|}
\hline Tratamento & Aplicação $^{1}$ & $\begin{array}{c}\text { Desfolha } \\
(\%)\end{array}$ & $\begin{array}{l}\text { PMG } \\
\text { (g) }\end{array}$ & $\begin{array}{l}\text { Produtividade } \\
\left(\mathrm{kg} \mathrm{ha}^{-1}\right)\end{array}$ \\
\hline Testemunha & - & $83,75 \mathrm{a}$ & $175,50 \mathrm{a}$ & $3558 \mathrm{a}$ \\
\hline Tetraconazol & V7 & $71,00 \mathrm{de}$ & $174,50 \mathrm{a}$ & $4506 \mathrm{a}$ \\
\hline Tetraconazol & V9 & $70,00 \mathrm{e}$ & $177,75 \mathrm{a}$ & $3648 \mathrm{a}$ \\
\hline Tetraconazol & $\mathrm{R} 2$ e $\mathrm{R} 5.1$ & $72,00 \mathrm{~d}$ & $178,00 \mathrm{a}$ & $3988 \mathrm{a}$ \\
\hline Tetraconazol & R5.1 & $73,75 \mathrm{c}$ & $168,00 \mathrm{a}$ & $4044 \mathrm{a}$ \\
\hline Pyraclostrobina+epoxiconazol & V7 & 70,75 de & $172,00 \mathrm{a}$ & $4222 \mathrm{a}$ \\
\hline Pyraclostrobina+epoxiconazol & V9 & $71,75 \mathrm{~d}$ & $172,25 \mathrm{a}$ & $3871 \mathrm{a}$ \\
\hline Pyraclostrobina+epoxiconazol & $\mathrm{R} 2$ e $\mathrm{R} 5.1$ & $66,75 \mathrm{f}$ & $170,75 \mathrm{a}$ & $3743 a$ \\
\hline Pyraclostrobina+epoxiconazol & R5.1 & $75,00 \mathrm{~b}$ & $168,75 \mathrm{a}$ & $4099 \mathrm{a}$ \\
\hline CV (\%) & & 1,15 & 6,37 & 10,60 \\
\hline
\end{tabular}


$\mathrm{Na}$ cultivar CD 205, elevado nível da doença foi observado em R5.3, chegando a testemunha a $62 \%$ de severidade (Tabela 3). Observou-se na avaliação prévia uma severidade de $12 \%$, caracterizando uma situação de elevado potencial de inóculo. A primeira área com a presença da ferrugem da soja na região de Palmeira, safra 2003/04, refere-se ao ensaio apresentado nas Tabelas 3 e 4 . Verifica-se que o controle químico é menos eficiente na redução da doença quando o fungo está presente na planta em elevada severidade. As aplicações realizadas no estádio fenológico R1 (início do florescimento) apresentaram melhores resultados de eficiência no controle do patógeno, sendo a severidade estatisticamente inferior à testemunha sem controle, para os dois produtos avaliados. À medida que se atrasou o controle, a severidade dos tratamentos foi semelhante à testemunha sem aplicação, independente do produto utilizado (Tabela 3). Para tanto, o momento da infecção da ferrugem nos estádios iniciais da soja sob condições climáticas favoráveis para o desenvolvimento do patógeno, poderão determinar a necessidade de controle. O que se sabe é que períodos contínuos de molhamento das folhas acima de seis horas, por chuva ou orvalho e temperaturas diárias variando de $15^{\circ} \mathrm{C}$ a $30^{\circ} \mathrm{C}$, favorecem o desenvolvimento da doença (BROMFIELD, 1984; MARCHETTI et al., 1976).

Durante o período de maior precipitação, meses de dezembro/2003, janeiro e março/2004, foi verificado que a evolução da doença foi mais pronunciada no ensaio em que coincidiu com o início da fase reprodutiva (R1) da soja no mês de janeiro/2004 (Tabela 3).

TABELA 3 - Severidade da ferrugem asiática sobre a cultivar CD 205, safra 2003/04, Palmeira-PR.

\begin{tabular}{|c|c|c|c|c|c|}
\hline Tratamento & & & & ação & \\
\hline & Aplicação ${ }^{1}$ & Prévia & $9 \mathrm{DAA}^{*}$ & $22 \mathrm{DAA}^{*}$ & $29 \mathrm{DAA}^{*}$ \\
\hline Testemunha & - & $12 \mathrm{a}$ & $19,25 \mathrm{a}$ & $23,00 \mathrm{a}$ & $62,00 \mathrm{a}$ \\
\hline Tetraconazol & R1 & $12 \mathrm{a}$ & $16,00 a b$ & $13,75 b c$ & $41,50 \mathrm{~b}$ \\
\hline Tetraconazol & R2 e R5.1 & $12 \mathrm{a}$ & $17,00 a b$ & $17,00 \mathrm{ab}$ & 63,75 a \\
\hline Tetraconazol & R5.1 & $12 \mathrm{a}$ & $21,25 \mathrm{a}$ & $22,00 \mathrm{a}$ & $52,50 \mathrm{ab}$ \\
\hline Pyraclostrobina+epoxiconazol & $\mathrm{R} 1$ & $12 \mathrm{a}$ & $14,25 \mathrm{~b}$ & $10,75 b$ & $38,75 \mathrm{~b}$ \\
\hline Pyraclostrobina+epoxiconazol & R2 e R5.1 & $12 \mathrm{a}$ & $21,25 \mathrm{a}$ & $17,50 a b$ & 65,25 a \\
\hline Pyraclostrobina+epoxiconazol & R5.1 & $12 \mathrm{a}$ & $21,50 \mathrm{a}$ & $23,00 \mathrm{a}$ & $54,75 \mathrm{ab}$ \\
\hline CV (\%) & & 0,0 & 14,44 & 16,58 & 16,06 \\
\hline
\end{tabular}

Médias seguidas pela mesma letra na vertical não diferem estatisticamente pelo teste de Tukey a $5 \%$ de probabilidade.

${ }^{1}$ Estádio fenológico.

* DAA: dias após a primeira aplicação.

Não foi observada diferença estatística na produtividade utilizando os diferentes ingredientes ativos, em aplicação única e duas aplicações, pelo teste de Tukey a $5 \%$ de probabilidade (Tabela 4 ). Os tratamentos iniciados em 16/01/2004 no início de florescimento (R1) e florescimento pleno (R2) apresentaram produtividade estatisticamente superior à testemunha. A aplicação iniciada em 15/02/2004 no início da formação de grãos (R5.1), quando a doença já estava estabelecida, mostrou produtividade semelhante à testemunha sem controle.

Foi observada redução de desfolha e aumento significativo do rendimento nos tratamentos com os ingredientes ativos tetraconazol e pyraclostrobina + epoxiconazol nas primeiras aplicações na situação de elevada severidade da doença, em R1 (Tabela 4). É possível que o resultado obtido não esteja relacionado com o estádio fenológico da planta e sim ao momento da aplicação em detrimento ao nível de severidade da doença. Á medida que a aplicação foi adiada em R2 e R5.1 foi verificado aumento significativo da doença. A diferença de produtividade observada na soja com uma aplicação em R1 foi de $1175 \mathrm{~kg} \mathrm{ha}^{-1}$ para o tratamento pyraclostrobina + epoxiconazol e $1155 \mathrm{~kg}$ $\mathrm{ha}^{-1}$ para o tratamento tetraconazol quando comparado à testemunha. 
REIS, E.F. et al. Controle químico da ferrugem asiática...

TABELA 4 - Percentagem de desfolha, peso de mil grãos e produtividade da cultivar CD 205, após aplicação de fungicidas em diferentes estádios fenológicos, safra 2003/04, Palmeira-PR.

\begin{tabular}{lcccc}
\hline Tratamento & Aplicação & $\begin{array}{c}\text { Desfolha } \\
(\%)\end{array}$ & $\begin{array}{c}\text { PMG } \\
(\mathrm{g})\end{array}$ & $\begin{array}{c}\text { Produtividade } \\
\left(\mathrm{kg} \mathrm{ha}^{-1}\right)\end{array}$ \\
\hline Testemunha & - & $90,50 \mathrm{a}$ & $130,00 \mathrm{~b}$ & $1664 \mathrm{~b}$ \\
Tetraconazol & $\mathrm{R} 1$ & $70,25 \mathrm{~d}$ & $145,00 \mathrm{a}$ & $2819 \mathrm{a}$ \\
Tetraconazol & $\mathrm{R} 2+\mathrm{R} 5.1$ & $75,00 \mathrm{c}$ & $145,75 \mathrm{a}$ & $2617 \mathrm{a}$ \\
& & & & $2137 \mathrm{ab}$ \\
Tetraconazol & $\mathrm{R} 5.1$ & $82,25 \mathrm{~b}$ & $140,50 \mathrm{a}$ & $2839 \mathrm{a}$ \\
Pyraclostrobina+epoxiconazol & $\mathrm{R} 1$ & $68,50 \mathrm{~d}$ & $145,25 \mathrm{a}$ & $2709 \mathrm{a}$ \\
Pyraclostrobina+epoxiconazol & $\mathrm{R} 2+\mathrm{R} 5.1$ & $74,00 \mathrm{c}$ & $145,00 \mathrm{a}$ & $2018 \mathrm{ab}$ \\
Pyraclostrobina+epoxiconazol & $\mathrm{R} 5.1$ & $81,75 \mathrm{~b}$ & $139,25 \mathrm{a}$ & 18,27 \\
CV (\%) & & 1,76 & 2,61 & $\mathrm{C}$ \\
\hline
\end{tabular}

Médias seguidas pela mesma letra na vertical não diferem estatisticamente pelo teste de Tukey a 5\% de probabilidade.

${ }^{1}$ Estádio fenológico

\section{CONCLUSÕES}

Verificou-se que o controle químico da ferrugem da soja, com base no estádio fenológico da cultura, não é um indicativo de controle eficiente, devido o patógeno estabelecer-se em qualquer estádio da planta durante o ciclo vegetativo. A aplicação de fungicidas, logo após o aparecimento dos primeiros sintomas com $0,16 \%$ de severidade na cultivar BRS 133 e, com a ferrugem já avançada, apresentando $12 \%$ de severidade na cultivar CD 205, favoreceu os melhores resultados de eficácia, indicando que quanto mais rápida for a identificação da doença na cultura mais eficiente é o controle. O controle químico através do triazol (tetraconazol) e da mistura de triazol + estrobilurina (pyraclostrobina + epoxiconazol), apresentaram controle eficiente do fungo favorecendo a redução do progresso da doença.

\section{REFERÊNCIAS}

1. FEHR, W.R.; CAVINESS, C.E. Stage of soybean development. Ames: lowa State University, 1981. 12 p. (Special report, 80).

2. GODOY, C.V., CANTERI, M.G. Efeitos protetor, curativo e erradicante de fungicidas no controle da ferrugem da soja causada por Phakopsora pachyrhizi, em casa de vegetação. Fitopatologia Brasileira, v. 28, n. 29, p. 97-101, 2004.

3. GODOY, C.V.; YORINORI, J.T. Ferrugem asiática. Revista Plantio Direto, n. 73, p. 19-20, 2003.

4. JACCOUD FILHO, D.S.; HIAR, C.P.; BONA, P.F.; GASPERINI, L. Ocorrência da ferrugem da soja na região dos Campos Gerais do Paraná. In: REUNIÃO DE PESQUISA DE SOJA DA REGIÃO CENTRAL DO BRASIL, 23., Londrina, 2001. Resumos. Londrina, 2001. p. 109.

5. ONO, Y., BURITICA, P.; HENNEN, J.F. Delimitation of Phakopsora, Physopella and Cerotelium and their species on Leguminosae. Mycological Research, n. 96, p. 825-850, 1992.

6. SINCLAIR, J.B.; HARTMAN, G.L. Soybean rust. In: HARTMAN, G.L.; SINCLAIR, J.B.; RUPE, J.C. (Eds.). Compendium of soybean diseases. 4. ed. St. Paul: American Phytopathological Society, 1999. p. 25-26.

7. YANG, X.B.; TSCHANZ, A.T.; DOWLER, W.M.; WANG, T.C. Development of yield loss models in relation to reductions of components of soybean infected with Phakopsora pachyrhizi. Journal of Phytopathology, n. 81, p. 1420-1426, 1991.

8. YORINORI, J.T.; PAIVA, W.M.; FREDERICK, R.D.; FERNANDEZ, P.F.T. Ferrugem da soja (Phakopsora pachyrhizi) no Brasil e no Paraguai, nas safras 2000/01 e 2001/02. In: CONGRESSO BRASILEIRO DE SOJA, 2002, Foz do Iguaçú, Anais. Foz do Iguaçú, 2002. p. 94. 
